\title{
Experiences with Self-Organizing, Decentralized Grids Using the Grid Appliance
}

\author{
David Isaac Wolinsky and Renato Figueiredo \\ University of Florida \\ (davidiw,renato)@acis.ufl.edu
}

\begin{abstract}
"Give a man a fish, feed him for a day. Teach a man to fish, feed him for a lifetime" - Lau Tzu

Large-scale grid computing projects such as TeraGrid and Open Science Grid provide researchers vast amounts of compute resources but with requirements that could limit access, results delayed due to potentially long job queues, and environments and policies that might affect a user's work flow. In many scenarios and in particular with the advent of Infrastructure-as-a-Service (IaaS) cloud computing, individual users and communities can benefit from less restrictive, dynamic systems that include a combination of local resources and on-demand resources provisioned by one or more IaaS provider. These types of scenarios benefit from flexibility in deploying resources, remote access, and environment configuration.

In this paper, we address how small groups can dynamically create, join, and manage grid infrastructures with low administrative overhead. Our work distinguishes itself from other projects with similar objects by enabling a combination of decentralized system organization and user access for job submission in addition to a web 2.0 interfaces for managing grid membership and automate certificate management. These components contribute to the design of the "Grid Appliance," an implementation of a wide area overlay network of virtual workstations (WOW), which has developed over the past six years into a mature system with several deployments and many users. In addition to an architectural description, this paper contains lessons learned during the development and deployment of "Grid Appliance" systems and a case study backed by quantitative analysis that verifies the utility of our approach.
\end{abstract}

\section{Categories and Subject Descriptors}

C.2.4 [Computer-Communication Networks]: Distributed Systems-Distributed applications

\section{General Terms}

Design

Permission to make digital or hard copies of all or part of this work for personal or classroom use is granted without fee provided that copies are not made or distributed for profit or commercial advantage and that copies bear this notice and the full citation on the first page. To copy otherwise, to republish, to post on servers or to redistribute to lists, requires prior specific permission and/or a fee.

HPDC'11, June 8-11, 2011, San Jose, California, USA.

Copyright 2011 ACM 978-1-4503-0552-5/11/06 ...\$10.00.

\author{
Keywords \\ Grid, Cloud, P2P Grid
}

\section{INTRODUCTION}

Grid computing presents opportunities to combine distributed resources to form powerful systems. Due to the challenges in coordinating resource configuration and deployment, researchers tend to either become members of existing grids or deploy their own private resources. The former approach is limited by lack of flexibility in the environment and policies, while the latter requires expertise in systems configuration and management. Though there exists a wealth of middleware available, including resource managers such as Condor [1], Torque (PBS) [2], and Sun Grid Engine [3], many see the cost of installing and managing these systems as being greater than their usefulness and as a result turn to inefficient ad hoc resource discovery and allocation. To combine resources across multiple domains solutions there exist solutions such as the Globus Toolkit [4] or gLite [5]; however, these tool sets come with their own challenges that require the level of expertise most researchers in fields outside of information technology lack.

With the recent advent of cost-effective on-demand computing through Infrastructure-as-a-Service "clouds", new opportunities for user-deployed grids have arisen; where, for example, a small local computer cluster can be complemented by dynamically provisioned resources that run "cloudburst" workloads. However, while cloud-provisioned resources solve the problem of on-demand instantiation, the problem of how to configure these resources to seamlessly and securely integrate with one's infrastructure remains a challenge. In particular, considering that users may provision resources from multiple IaaS providers, the configuration demands are similar to a distributed grid: while a cloud image can be encapsulated with a grid computing stack, it still needs configuration in terms of allocating and distributing the appropriate certificates, network configuration to establish end-to-end connectivity, and proper configuration of the middleware to establish worker, submit, and scheduler nodes.

In this paper, we present techniques that reduce the entry barrier in terms of necessary expertise and time investment in deploying and extending ad hoc, distributed grids. To verify this assertion, we have implemented a system supporting these ideas in the "Grid Appliance," which as will be demonstrated, allows users to focus on making use of a grid while minimizing their efforts in setting up and manag- 
ing the underlying components. The core challenges solved by our approach include:

- decentralized directory service for organizing grids,

- decentralized job submission,

- grid single sign on through web services and interfaces,

- sandboxing with network support,

- and all-to-all connectivity despite network asymmetries.

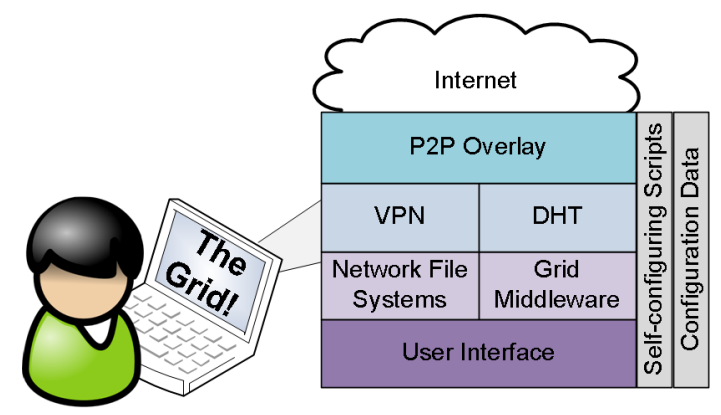

Figure 1: The "Grid Appliance" connects to other resources over a common network. Both the grid middleware and the VPN use the P2P overlay to configure and connect the user to other members of the grid. The process uses configuration data provided by the web interface to self-configure the system using information available in the P2P network.

The "Grid Appliance" project and concepts have been actively developed and used in several projects for the past six years. Of these projects, Archer, a distributed grid for computer architecture research, has demonstrated the feasibility and utility of this approach by deploying a shared collaborative infrastructure spanning clusters across six US universities, where the majority of the nodes are constrained by network address translation (NAT). Every resource in Archer is configured in the same, simple manner: by deploying a "Grid Appliance" that self-configures to join a widearea grid. Researchers interested or desiring the ability to access both grid resources and specialized commercial simulation tools (such as Simics) can easily use and contribute resources from this shared pool with little effort by joining a website, downloading a configuration image and a virtual machine (VM), and starting the VM inside a VM manager (VMM). Upon completion of the booting process, users are connected to the grid and able to submit and receive jobs.

At the heart of our approach lies a P2P infrastructure based upon a distributed hash table (DHT) useful for decentralized configuration and organization of systems. Peers are able to store key, value pairs into the DHT and to query the DHT with a key and potentially receive multiple values efficiently. The DHT provides discovery and coordination primitives for the configuration of a decentralized $\mathrm{P} 2 \mathrm{P}$ virtual private network (VPN), which supports unmodified applications across a network overlay. The DHT is also used for the decentralized coordination of the grid. Users can configure their grid through a web interface, which outputs configuration files that can be used with the "Grid Appliance."
The techniques described in this paper have many applications. The basic system supports the creation of local grids by starting a virtual machine on the computers intended for use within the grid and using LAN multicast for discovery. It allows users to seamlessly combine their dedicated grids with external resources such as workstations and cloud resources. The level of familiarity with security, operating systems, and networking is minimal as all the configuration details are handled as components of the system. Management of the system including users and network configuration utilizes a social networking like group interface, while deployment uses pre-built virtual machine images. A graphical overview of the system is illustrated in Figure 1.

These techniques simplify the tethering of resources across disparate networks The setup of security, connectivity, and their continuous management imposes considerable administrative overhead, in particular when networks are constrained by firewalls and NAT devices that prevent direct communication with each other, and which are typically outside the control of a user or lab. Our approach integrates decentralized systems behind NATs in a manner that does not require the setup of exceptions and configuration at NAT/firewall by system administrators.

The rest of the paper is as follows. Section 2 highlights our previous work to provide background for our contributions in this paper. In Section 3, we describe the components of our "Grid Appliance" WOW. Section 4 provides a case study of a grid deployment using standard grid deployment techniques compared to our "Grid Appliance," describing qualitatively the benefits and evaluating quantitatively the overheads of this approach. We share our experiences from this long running project in Section 5. Finally, Section 6 compares and contrasts other solutions to these problems.

\section{WOWS}

This work furthers the vision began by our earlier work wide-area overlay of virtual workstations [6] (WOW). The WOW paper established the use of virtualization technologies, primarily virtual networking and virtual machines, to support dynamic allocation of additional resources in grids that span wide area networks. For reference, the extensions made in this paper to the WOW concept are means for the dynamic creation of grids with support for security, decentralized access, and user-friendly approaches to grid management. This section covers the development of WOWs over the years as it relates to our other publications and as means to distinguish the contributions made in this paper.

\subsection{P2P Overlays}

Peer-to-peer or P2P systems create environments where members have a common functionality. P2P systems are often used for discovery in addition to some user-specific service, such as voice and video with Skype or data sharing with BitTorrent. Many forms of P2P have autonomic features such as self-healing and self-optimization with the ability to support decentralized environments. As we will show, this makes their application in our system very attractive.

For the "Grid Applianc," we have chosen to use Brunet [7], a type of structured overlay. Structured overlays tend to be used to construct distributed hash tables (DHT) and in comparison to unstructured overlays provide faster guaranteed search times $(O(\log N)$ compared to $O(N)$, where $\mathrm{N}$ is the 
size of the network). The two most successful structured overlays are Kademlia [8], commonly used for decentralized BitTorrent, and Dynamo [9], to support Amazon's web site and services.

Brunet support for NAT traversal makes it unique from other structured overlays. Originally in the WOWs [6], Brunet facilitated the dynamic connections amongst peers in the grid. Since then, it has been extended to support DHT with atomic operations [10], efficient relays when direct NAT traversal fails [11], resilient overlay structure and routing [12], and cryptographically secure messaging [11].

\subsection{Virtual Private Networks}

A common question with regards to this work is "why VPNs?" The core reason is connectivity. IPv4 has a limited address space, which has been extended through the use of NAT allowing a single IP to be multiplexed by multiple devices. This creates a problem; however, as it breaks symmetry in the Internet limiting the ability for certain peers to become connected and which peers can initiate connections. With the advent of IPv6, the situation might improve, but there are no guarantees that NATs will disappear nor can users be certain that firewalls will not be in place that inhibit symmetry. A VPN circumvents these issues, so long as the user can connect to the VPN, as all traffic is routed through a successfully connected pathway.

The problem with traditional VPN approaches is management overhead including maintaining resources on public IP addresses and establishing links amongst members in the VPN. The VPN used in the system is called IPOP $[11,13]$. IPOP (IP over P2P), as the name implies, uses a P2P overlay (Brunet) to route IP messages. By using P2P, maintaining dedicated bootstrap nodes have less overhead, our approach with IPOP allows an existing Brunet infrastructure to bootstrap independent Brunet infrastructures in order to isolate IPOP networks in their own environments [14].

Once IPOP has entered its unique Brunet overlay, it obtains an IP address. IP address reservation and discovery relies on Brunet's DHT. Each VPN stores its P2P identifier into the DHT at the generated by the desired IP address, such that the key, value pair is (hash $(I P), P 2 P)$. In order to ensure there are no conflicts, the storing of this value into the DHT uses an atomic operation, which succeeds only if no other peer has stored a value int hash $(I P)$.

The process for creating connections begins when IPOP receives an outgoing message. First it parses the destination address and queries the DHT for the remote peers $\mathrm{P} 2 \mathrm{P}$ address. The peer then attempts to form a secure, direct connection with the remote peer using Brunet's secure messaging layer. Once that has formed, packets to that IP address are directed over that secure link.

In our original design [15], the virtual network was secured through a kernel-level IPsec stack, a model kept through our first generation Archer deployment. This approach only secures virtual network links between parties and does not secure the $\mathrm{P} 2 \mathrm{P}$ layer; furthermore, in IPsec configuration each peer requires a unique rule for every other peer, which limited the maximum number of peers in the VPN. Securing the P2P layer is important, otherwise malicious users could easily derail the entire system, but securing with IPsec would practically negate the benefits of the P2P system, because of network configuration issues related to NATs and firewalls. In our modern deployments, we have employed the security layer at the $\mathrm{P} 2 \mathrm{P}$ layer, which in turn also secures virtual networking links.

For grids that rely upon VPNs to connect resources and users, this can impose the need for a certificate for the VPN and one for the grid. Though in our approach, we avoid this problem by using a VPN that allows a user to verify the identity of a remote peer and obtain its certificate, and have taken advantage of hooks in grid software that are called to verify a remote peers authenticity. In other words, user access is limited by the VPN and identity inside the grid is maintained by that same certificate. This might not be possible if all users were submitting from the same resources but is feasible in our system since each user submits from their own system.

\subsection{Virtual Machines in Grid Computing}

Earlier work [16] advocated the use of virtual machines (VMs) in grid computing for improved security and customization. Others since $[17,18,19]$ have been established VMs as means for sandboxing, that is environments that allow untrusted users to use trusted resources in a limited fashion. VMs run as a process on a system, where processes running inside the VM have no access to the host operating system. Furthermore, VMs can have limited or no networking access as controlled by the host, which effectively seals them in a cage or sandbox protecting the hosts environment. VMs are also useful for customization and legacy applications, since a developer can configure the VM and then distribute it as an appliance, with the only requirement on the end user being that they have a VM software or manager. Quantitatively, previous work has shown that CPU-bound tasks perform fairly well running with no more than $10 \%$ overhead and in some cases $0 \%$, which is the case with VMs like Xen.

While not a direct correlation to grid computing, clouds have benefited significantly from VMs. VMs are the magic behind cloud infrastructures that provide IaaS, such as EC2. In these environments, users are able to create customized instances, or packaged operating systems and applications, inside of cloud environments, share with each other, and dynamically create or shutdown them as necessary. While the application of clouds is generic, it can easily be applied towards grids. A user can create push excess jobs into the cloud, when there is overflow, high demands, or the user does not want to maintain their own hardware. One challenge, however, is the dynamic creation of a grid as well as extension of an existing grid using the cloud, challenges that are addressed in this paper.

\section{ARCHITECTURAL OVERVIEW}

Our approach attempts to reuse as many available components to design a grid middleware generic enough that th ideas can be applied to other middleware stacks. As a result, our contribution in this paper and in particular this section focuses primarily on the following key tasks: making grid construction easy, supporting decentralized user access, sandboxing the users environment, limiting access to the grid to authorized identities, and ensuring priority on users own resources.

\subsection{Web Interface and the Community}

Before deploying any software or configuring any hardware, a grid needs organization including certificate manage- 


\begin{tabular}{|c|c|c|c|c|}
\hline & Description & Scalability & $\begin{array}{ll}\begin{array}{l}\text { Job queue / submission } \\
\text { site }\end{array} & \\
\end{array}$ & API Requirements \\
\hline Boinc & $\begin{array}{l}\text { Volunteer computing, ap- } \\
\text { plications ship with Boinc } \\
\text { and poll head node for } \\
\text { data sets }\end{array}$ & $\begin{array}{l}\text { Not explicitly mentioned, } \\
\text { limited by the ability of the } \\
\text { scheduler to handle the de- } \\
\text { mands of the client }\end{array}$ & $\begin{array}{l}\text { Each application has a dif- } \\
\text { ferent site, no separation } \\
\text { from job queue and sub- } \\
\text { mission site }\end{array}$ & $\begin{array}{l}\text { Applications are bundled } \\
\text { with Boinc and must be } \\
\text { written to use the Boinc } \\
\text { API in order to retrieve } \\
\text { data sets and submit re- } \\
\text { sults to the head node }\end{array}$ \\
\hline BonjourGriq & $\begin{array}{l}\text { Desktop grid, use zeroconf } \\
\text { / Bonjour to find available } \\
\text { resources in a LAN }\end{array}$ & $\begin{array}{l}\text { No bounds tested, lim- } \\
\text { its include multicasting } \\
\text { overheads and processing } \\
\text { power of job queue node }\end{array}$ & $\begin{array}{l}\text { Each user has their own } \\
\text { job queue / submission site }\end{array}$ & None \\
\hline Condor & $\begin{array}{l}\text { High throughput comput- } \\
\text { ing / on demand / desktop } \\
\text { / etc / general grid com- } \\
\text { puting }\end{array}$ & Over $10,000^{1}$ & $\begin{array}{l}\text { Global job queue, no limit } \\
\text { on submission sites, sub- } \\
\text { mission site communicates } \\
\text { directly with worker nodes }\end{array}$ & $\begin{array}{l}\text { Optional API to support } \\
\text { job migration and check } \\
\text { pointing }\end{array}$ \\
\hline PastryGrid & $\begin{array}{l}\text { Use structured overlay } \\
\text { Pastry to form decentral- } \\
\text { ized grids }\end{array}$ & $\begin{array}{l}\text { Decentralized, single node } \\
\text { limited by its processing } \\
\text { power, though collectively } \\
\text { limited by the Pastry DHT }\end{array}$ & $\begin{array}{l}\text { Each connected peer main- } \\
\text { tains its own job queue and } \\
\text { submission site }\end{array}$ & None \\
\hline $\begin{array}{l}\text { PBS } \\
\text { Torque [2] }\end{array}$ & $\begin{array}{l}\text { Traditional approach to } \\
\text { dedicated grid computing }\end{array}$ & up to $20,000 \mathrm{CPUs}^{2}$ & $\begin{array}{l}\text { Global job queue and sub- } \\
\text { mission site }\end{array}$ & None \\
\hline SGE & $\begin{array}{l}\text { Traditional approach to } \\
\text { dedicated grid computing }\end{array}$ & $\begin{array}{l}\text { Tested up to } 63,000 \text { cores } \\
\text { on almost } 4,000 \text { hosts }^{3}\end{array}$ & $\begin{array}{l}\text { Global job queue and sub- } \\
\text { mission site }\end{array}$ & None \\
\hline XtremWeb & $\begin{array}{l}\text { Desktop grid, similar to } \\
\text { Condor but uses pull in- } \\
\text { stead of push, like Boinc }\end{array}$ & $\begin{array}{l}\text { Not explicitly mentioned, } \\
\text { limited by the ability of the } \\
\text { scheduler to handle the de- } \\
\text { mands of clients }\end{array}$ & $\begin{array}{l}\text { Global job queue, separate } \\
\text { submission site, optionally } \\
\text { one per user }\end{array}$ & $\begin{array}{l}\text { No built-in support for } \\
\text { shared file systems }\end{array}$ \\
\hline
\end{tabular}

\section{Table 1: Grid Middleware Comparison}

ment, grid access, user account management, and delegation of responsibilities. These are complex questions, which can be challenging to address, though for less restrictive systems, like a collection of academic labs sharing clusters, they may be very easy. One of the professors could handle the initial authorization of all the other labs and then delegate to them the responsibility of allowing their affiliates, such as students and scholars access.

For academic environments, grids become more challenging when the professor or worse yet students must maintain the certificates, handling certificate requests, and placing signed certificates in the correct location. Our solution to this potentially confusing area was a group interface, akin to something like Facebook's or Google's groups. Albeit, those types of groups are not hierarchal, which is a necessity in order to have delegated responsibilities. Thus we have a two layer approach, a grid group for members of the grid trusted by the grid organizers and user groups for those who are trusted by those in the grid group. Members of the grid group can create their own user groups. A member of a user group can gain access to the grid by downloading grid configuration data available within the user group web interface. This configuration data comes in the format of a disk image, when added to a "Grid Appliance" VM, it is used to obtain the user's credentials and enabling them to connect to the grid.

To give an example, consider our computer architecture grid, Archer. Archer was seeded initially by the University of Florida, so we are the founders and maintainers of the Archer grid group. As new universities and independent researchers have joined Archer, they request access to this group. Upon receiving approval, they then need to form their own user group so that they can allow others to connect to the grid. So a trusted member might create a user group titled "Archer for University X" and all members of university $\mathrm{X}$ will apply for membership in that group. The creator can make decisions to either accept or deny these users. Once the user has access, they will download their configuration data formatted as a virtual disk image and the "Grid Appliance" VM and start the "VM." After starting the VM, the user will be connected to the grid and able to submit and receive jobs.

Joining is easy; a grid requires a user to sign onto a website and download a configuration data, which can then be used on multiple systems. To support this process, the configuration data contains cryptographic information that facilitates acquisition of a signed certificate from the web interface through XML-RPC over HTTPS. The process begins by either booting the "Grid Appliance" or restarting a "Grid Appliance" service. When starting the service will detect if there is new configuration data, and if there is, it contacts the web interface with the cryptographic information and a public key. The web interface verifies the user's identity, retrieves their profile from its database and binds that information with the public key to create a certificate request, which will then be signed and returned to the user.

With a public web interface, we have been able to create a variety communities. One of particular interest is not the grid itself but rather a bootstrapping community for grids. The web interface has been designed to support many grid groups, so too has the P2P infrastructure as it supports bootstrapping into unique private overlays for individual grids by means of Brunet's ability to support recursive bootstrapping. By using the public interface, users have an opportunity to reuse our bootstrap infrastructure and only need to focus on the configuration of their VPN

\footnotetext{
${ }^{1}$ http://www.cs.wisc.edu/condor/CondorWeek2009/ condor \_presentations/sfiligoi-Condor \_WAN $\backslash$ -scalability.pdf

${ }^{2}$ http://www. clusterresources. com/docs/211

${ }^{3}$ http://www. sun.com/offers/docs/Extremel _Scalability\_SGE.pdf
} 


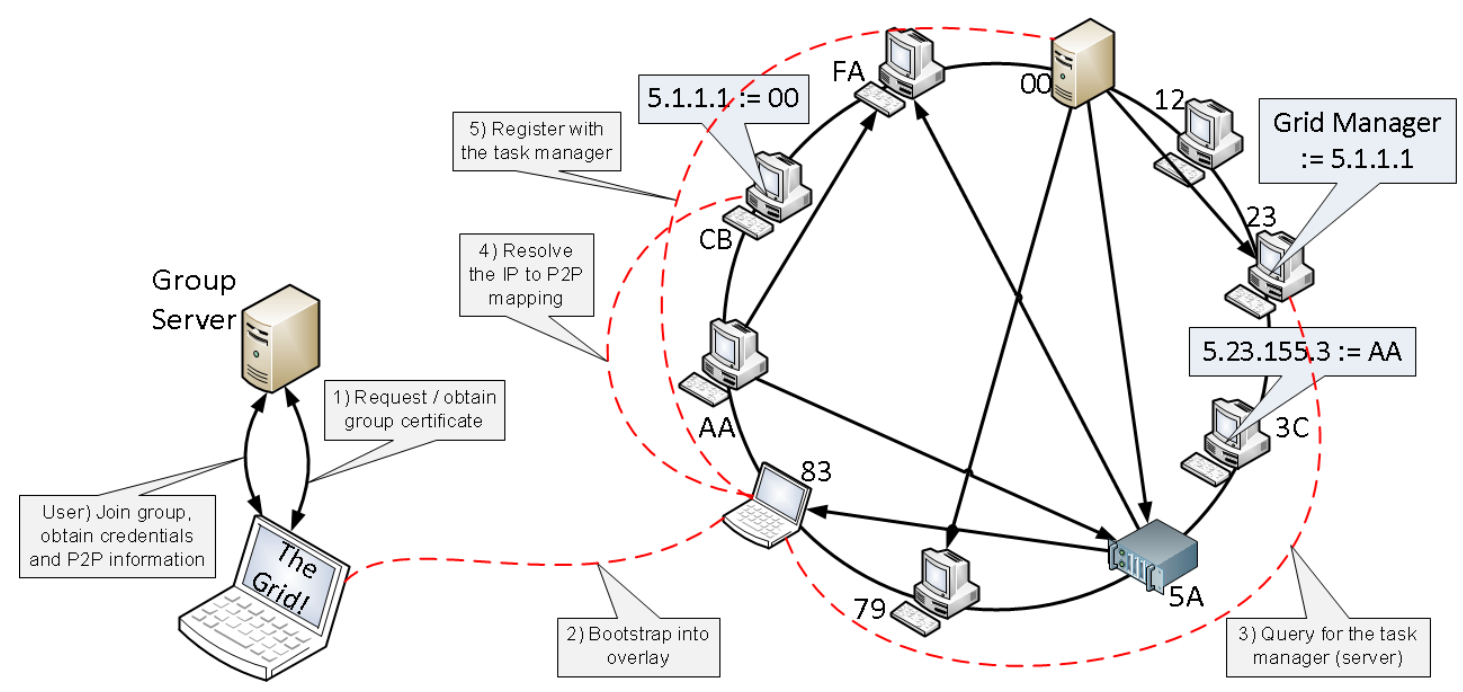

Figure 2: An example deployment scenario: obtaining configuration files, starting the appliance, and connecting with a resource manager.

and grid services, which has been trivialized to accepting or denying users access to a group and turning on resources. We would like to note that there is no need to make an explicit public grid community through the web interface, since all "Grid Appliances" come with a default configuration file that will connect them to an insecure public grid.

\subsection{The Organization of the Grid}

The previous section focused facilitation of grid configuration using the web interface and skirted the issues of detailed configuration and organization. The configuration of the grid mirrors that of the connection process. The first tier group maps to a common grid and each grid maps to a VPN. Thus when a user creates a new grid group, they are actually configuring a new VPN, which involves address range, security parameters, user agreements, and the name of the group. The system provides defaults for address range and security parameters, so users can focus on high level details like the user agreement and the grid's name.

As mentioned earlier, the second tier of groups enables members in the grid group to provide access to their community. It is also the location that users download their configuration data. The configuration files come in three flavors: submission, worker, or manager. Worker nodes strictly run jobs. Submission nodes can run jobs as well as submit jobs into the grid. Manager nodes are akin to head nodes, those that manage the interaction between worker and submission nodes.

While the configuration details are handled by the web interface and scripts inside the "Grid Appliance," organization of the grid, more specifically the linking of worker and submission nodes to manager nodes, relies on the DHT. Managers store their IP addresses into the DHT at the key managers. When workers and clients join the grid, they automatically query this key, using the results to configure their grid software. Managers can also query this key to learn of other managers to coordinate with each other.

\subsubsection{Selecting a Middleware}

Our grid composition is largely based upon a desire to support a decentralized environment, while still retaining reliability and limiting our documentation support efforts. As there exist many middlewares to support job submission and scheduling, we surveyed available and established middleware to determine how well they matched our requirements. Our results are presented in Table 1, which covers most of the well established middleware and some recent research projects focused on decentralized organization.

Of the resource management middlewares surveyed, we chose to use Condor as it matches closest with our goals due to its decentralized properties and focus on desktop grids. With Condor, we are able to have multiple submission points, a non-trivial obstacle in some of the other systems. Additionally, adding and removing resources in Condor can be done without any configuration from the managers. Conversely, in SGE and Torque, after resources have been added into the system, the administrator must manually configure the manager to control them. Most scheduling software assumes that resources are dedicated, while Condor supports opportunistic cycles, by detecting the presence of other entities and will suspend, migrate, or terminate a job, thus enabling desktop grids. A common drawback to established middlewares is the requirement of a manager node; having no manager in an ad hoc grid would be ideal.

\subsubsection{Self-Organizing Condor}

While the requirement of a central manager may be undesirable, they can easily be run inside a VM and Condor supports the ability to run many in parallel through the use of "flocking [20]." Flocking allows submission sites to connect to multiple managers. This serves two purposes: 1) to provide transparent reliability by supporting multiple managers and 2) users can share their resources through their own manager. Flocking allows each site to run its own manager or share the common manager.

To configure Condor, manager IP addresses are stored into the DHT using the key managers. Joining peers query the DHT to obtain a list of managers, selecting one randomly to use as its primary manager with the result used for flocking. If the system prefers managers from its group, it will randomly contact each manager in an attempt to find a match, selecting one at random if no match is found. Until a man- 
ager is found, the process repeats every 60 seconds. Upon finding a manager, the state of the system is verified every 10 minutes and new managers are added to the flock list.

\subsubsection{Putting It All Together}

The following summarizes the configuration and organization of the grid. Minimally a grid will constitute a manager, some workers, and a submitter. Referencing Figure 2 step "1," during system boot, without user interaction, each machine contacts the group website to obtain a valid VPN certificate. Whereupon, it connects to the $\mathrm{P} 2 \mathrm{P}$ overlay whose bootstrap peers are listed inside the configuration file, "step 2." At which point, the machine starts the VPN service running on top of the P2P overlay, also part of step "2." The self-configuring VPN creates a transparent layer hiding from the user and administrators the complexity in setting up a common fabric that can handle potential network dynamics. Machines automatically obtain a unique IP address and find their place inside the grid. For a manager machine, this means registering in the DHT (not shown), while clients and workers search for available managers by querying the DHT, step "3;" IPOP translates the IP to a P2P address, step "4;" and then client contacts the manager directly, step "5."

\subsection{Sandboxing Resources}

As tasks can run on worker and potentially submission nodes, we have devised means to sandbox the environments that do not limit user interactions with the system. While more traditional approaches to sandboxing emphasize a separation between worker and submission machine, in our deployments, very few users explicitly deploy worker machines, most are submission machines. Thus we developed our sandboxing techniques to limit the ability of submitted jobs on systems that are simultaneously being used for submission. So our sandboxing technique considers more than just locking down the machine but also ensuring a reasonable level of access.

\subsubsection{Securing the Resources}

The core of our sandboxing approach is to limit attacks to software in the system and not poorly configured user space, such as poorly chosen passwords or resources external to the "Grid Appliance." All jobs are run as a set of predefined user identities. When the jobs are finished executing, whether forcibly shutdown or completed successfully, all processes from that user are shutdown, preventing malicious trojan attacks. Those users only have access to the working directory for the job and those with permission for everybody. Escalation of privilege attacks due to poor passwords are prevented by disallowing use of "su" or "sudo" for these users. Finally, network access is limited to the VPN, thus they are unable to perform denial of service attacks on the Internet.

Additionally, systems can be configured such that the only network presented to them is that of the virtual network. To support this, IPOP has been enhanced to support a router mode, which can be bridged to a virtual machine adapter running on the host machine that connects to the network device running inside the VM. Not only does this improve performance, due to reduced I/O overhead, the same virtual network router can be used for multiple VMs.

To ensure that submit machines still have a high level of functionality without risking the system to external attacks even from users on the same network, user services are run only on a "host-only" network device within the virtual machine. This includes an SSH server and a Samba or Windows File Share. The user name matches that from the website, while the password defaults to "password." We would like to note that file sharing services work the opposite to that of host to guest as most VMs already have in place. Instead users can access their files on the VM from the host. This was done to limit potential attacks on submission machine.

\subsubsection{Respecting the Host}

Another aspect of sandboxing is respecting the usage of the host. While Condor can detect host usage on a machine it is running, when run inside a VM it cannot detect usage on the host. Thus it is imperative to support such a configuration otherwise our approach would be limited in that it can only be run during idle times. In the "Grid Appliance", this is addressed by running a light-weight agent on the host that communicates to the VM through the second Ethernet interface. The agent discovers a VM through multicast service discovery executed only on "host-only" virtual network devices. When a user accesses the host, the agent notifies a service in the VM, which results in running tasks being suspended, migrated, or terminated. The machine remains off limits until there has been no user activity for 10 minutes.

\subsubsection{Decentralized Submission of Jobs}

From the administrator's perspective, not requiring a submission machine is also a form of sandboxing. Maintaining a worker machine requires very low overhead, since jobs and their associated files are removed upon the completion of a job and corrupted workers can be deleted and redeployed. Maintaining a submission machine means user accounts, network access, providing data storage, and trusting users to play nicely on a shared resource. So having users be able to submit from their own resources reduces the overhead in managing a grid. It does come with a consequence, most grids provide shared file systems, which are statically mounted in all nodes. In a dynamic grid that might have multiple shares, this type of approach may not be very feasible.

All is not lost, for example, Condor provides data distribution mechanisms for submitted jobs. This can be an inconvenience, however, if only a portion of the file is necessary, as the entire file must be distributed to each worker. This can be particularly true with disk images used by computer architecture simulations and applications built with many modules or documentation. To support sparse data transfers and simplify access to local data, each "Grid Appliance" has a local NFS share exported with read-only permission. To address the issue of mounting a file system, there exists a tool to automatically mount file systems, autofs. autofs tool works by intercepting file system calls inside a specific directory, parsing the path, and mounting a remote file system. In the "Grid Appliance," accessing the path /mnt/ganfs/hostname, where hostname is either the IP address or hostname of an appliance, will automatically that appliance's NFS export without the need for super-user intervention. Mounts are automatically unmounted after a sufficient period of time without any access to the mounted file system. 


\section{DEPLOYING A CAMPUS GRID}

We now present a case study exploring a qualitative and quantitative comparison in deploying a campus grid and extending it into the "Cloud" using traditional techniques versus a grid constructed by "Grid Appliance." One of the target environments for the "Grid Appliance" is resources provided in distributed computer labs and many small distributed clusters on one or more university campus as shown in Figure 3. The goals in both these cases are to use commodity software, where available, and to provide a solution that is both simple but creates an adequate grid. In both cases, Condor is chosen as the middleware, which is a push scheduler and by default requires that all resources be on a common network thus a VPN will be utilized. Additionally, in this section, we cover details of the "Grid Appliance" that did not fit in the context of previous discussions in the paper.

\subsection{Background}

In this case study, we will compare and contrast the construction of two types of grids: a static grid configured by hand and a dynamic grid configured by the "Grid Appliance." Each grid is initially constructed using resources at the University of Florida and later extended to Amazon's EC2 and Future Grid at India University using Eucalyptus. Each environment has a NAT limiting symmetric communication: University of Florida resources are behind two layers, first an "iptables" NAT and then a Cisco NAT; EC2 resources have a simple 1:1 NAT; and the Eucalyptus resources appear to have an "iptables" NAT.

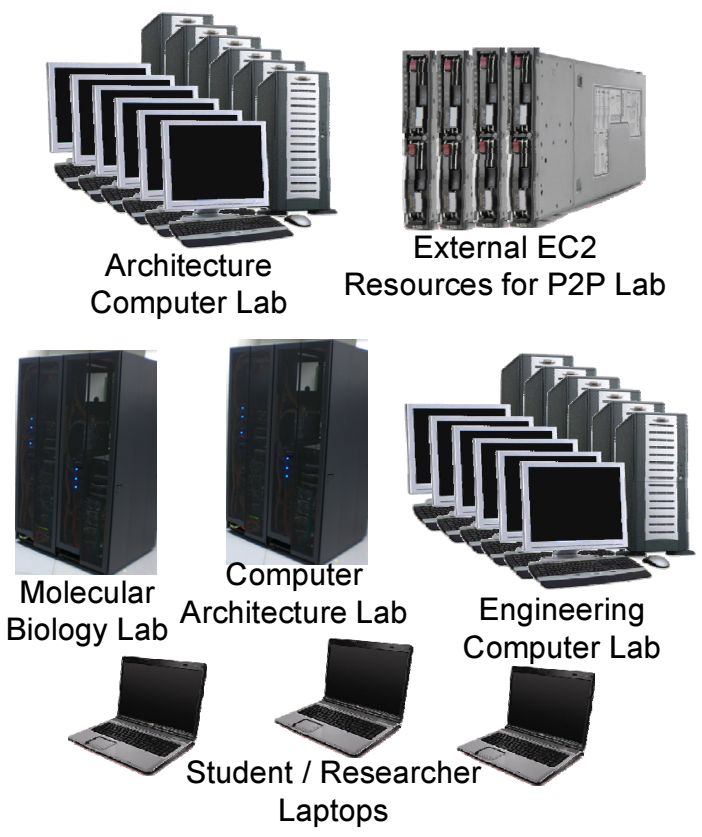

Figure 3: A collection of various computing resources at a typical university.

\subsection{Traditional Configuration of a Campus Grid}

A VPN must be used to connect the resources due to the lack of network symmetry across the sites. There exists a wealth of VPNs available [21, 22, 23] and some explicitly for grids $[24,25,26]$. For simplicity sake, OpenVPN was chosen due to the simplicity in its configuration. In reality, OpenVPN makes a poor choice because it is centralized, thus all traffic between submitter and worker must traverse the VPNs server. Whereas others in the list are distributed and thus allow nodes to communicate directly, but in order to do so, manual setup is required, a process, that would overwhelm many novice grid deployers. In all these cases, the VPN requires that at least a single node have a public address, thus we had to make a single concession in the design of this grid, that is, the OpenVPN server runs on a public node.

In order to connect to OpenVPN, it must know the server's address and have a signed certificate. While typically, most administrators would want a unique private key for each machine joining the grid, in our case study and evaluation, we avoided this process and used a common key, certificate pair. In doing so, there are potential dangers, for example, if any of the machines were hijacked, the certificate would have to be revoked and all machines would be rendered inoperable. To create a properly secured environment, each resource would have to generate or be provided a private key, a certificate request submitted to the certificate authority, and a signed certificate provided to the resource.

With the networking and security components in place, the next step is configuring grid middleware. Prior to deploying any resources, the manager must be allocated and its IP address provider to other resources in the system. Submission points are not a focus on this case study, though in general most systems of this nature have a single shared submission site. The challenges in supporting multiple submission points in this environment include creating certificates same as worker nodes, requiring users to configure OpenVPN and Condor, and handling NFS mounts. Whereas having a single submission point creates more work for the system administrator as mentioned earlier. Both approaches have their associated costs and neither is trivial. The evaluation assumes a single user submitting from a single resource.

To address potential heterogeneity issues. An administrator would need to collaborate with others to ensure that all resources are running a common set of tools and libraries. Otherwise an application that works well on one platform could cause a segmentation fault on another, through no fault of the user, but rather due to library incompatibilities.

To export this system into various clouds, an administrator starts by running an instance that contains their desired Linux distribution and then installing the grid utilities like Condor and OpenVPN. Supporting individualization of the resources is challenging. The simplest approach is to store all the configuration in that instance including the single private key, certificate pair as well as the IP address of the manager node. Alternatively, the administrator could build an infrastructure that receives certificate requests and returns a certificate. The IP address of the manager node and of the certificate request handler could be provided to the cloud via user data, a feature common to most IaaS clouds that allows users to provide either text or binary data that is available via a private URL inside a cloud instance.

\subsection{Grid Appliance in a Campus Grid}

All these configuration issues are exactly the reasons why "Grid Appliance" and its associated group Web interface are desirable for small and medium scale grids. The first 
component is deciding which web interface to use, public (www.grid-appliance.org) or private hosted on their own resources. Similarly, users can deploy their own P2P overlay or use our shared overlay.

The web interface enforces unique names for both the users and the groups. Once the user has membership in the second tier of groups, they can download a file that will be used to automatically configure their resources. As mentioned earlier, this handled obtaining a unique signed certificate, connecting to the VPN, and discovering the manager in the grid. Configuration of the VPN and grid are handled seamlessly, the VPN automatically establishes direct links with peers on demand and peers configure based upon information available in the P2P overlay dynamically allowing for configuration changes.

Heterogeneity is a problem that will always exist if individuals are given governance of their own resources. Rather than fight that process, the "Grid Appliance" approach is to provide a reference system and then include that version and additional programs in the resource description exported by Condor. Thus a user looking for a specific application, library, or computer architecture can specify that in their job description. Additionally, by means of the transparent NFS mounts, users can easily compile their own applications and libraries and export them to remote worker nodes.

Extending the "Grid Appliance" system into the clouds is easy. The similarity between a VM appliance and a cloud instance are striking. The only difference from the perspective of the "Grid Appliance" system is where to check for configuration data. Once a user has created a "Grid Appliance" in a cloud, everyone else can reuse it and just supply their configuration data as the user data during the instantiation of the instances. As we describe in Section 5.2, creating "Grid Appliance" from scratch is a trivial procedure.

As described in detail earlier, an administrator needs to install the necessary software either by deploying VMMs and VM appliances or installing "Grid Appliance" packages on Debian / Ubuntu systems. Additionally, these systems need to be packaged with the configuration files or floppy disk images. At which point, the systems will automatically configure and connect to the grid. An administrator can verify this by monitoring Condor. Additional resources can be added seamlessly, likewise resources can be removed by shutting them off without direct interaction with the "Grid Appliance" or manager node.

\subsection{Comparing the User Experience}

In the case of a traditional grid, most users will contact the administrator and make a request for an account. Upon receiving confirmation, the user will have the ability to SSH into a submission site. Their connectivity to the system is instantaneous, their jobs will begin executing as soon as it is their turn in the queue. User's will most likely have access to a global NFS. From the user's perspective, the traditional approach is very easy and straightforward.

With the "Grid Appliance," a user will obtain an account at the web interface, download a VM and a configuration file, and start the VM. Upon booting, the user will be able to submit and receive jobs. To access the grid, users can either SSH into the machine or use the consoles in the VM. While there is no single, global NFS, each user has their own unique NFS and must make their job submission files contain their unique path. For the most part, the user's perspective of the "Grid Appliance" approach has much of the same feel as the traditional approach. Although users have additional features such as accessing their files via Samba and having a portable environment for doing their software development.

\subsection{Quantifying the Experience}

The evaluation of these environments focuses on the time taken to dynamically allocate the resources, connect to the grid, and submit a simple job to all resources in the grid. In both systems, a single manager and submission node were instantiated in separate VMs. In the traditional setup, OpenVPN is run from the manager node. Each component in the evaluation was run three times. Between iterations, the submission node and the manager node were restarted to clear any state.

The times measured include the time from when the last grid resource was started to the time it reported to the manager node, Figure 4, as well as the time required for the submit node to queue and run a 5 minute job on all the connected workers, Figure 5. The purpose of the second test is to measure the time it takes for a submission site to queue a task to all workers, connect to the workers, submit the job, and to receive the results; thus a stress test on the VPN's ability to dynamically create links and verifying all-to-all connectivity. The tests were run on 50 resources (virtual machines / cloud instances) in each environment and then on a grid consisting of all 150 resources with 50 at each site.

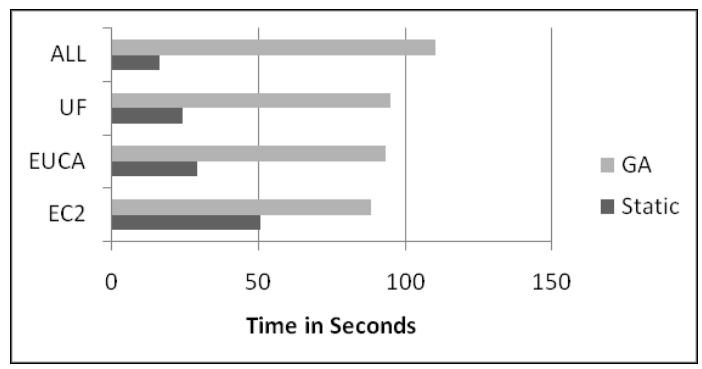

Figure 4: Comparison of times to construct a grid in various environments using both a statically configured grid and a grid constructed by the "Grid Appliance." Legend: EC2 - Amazon's EC2, Euca Indiana University's Eucalyptus, UF - University of Florida's ACIS Lab resources, Static - OpenVPN, GA - Grid Appliance.

In the previous section, we qualified why the approach was easier than configuring a grid by hand, though by doing so we introduce overheads related to configuration and organization. The evaluation verifies that these overheads do not conflict with the utility of our approach. Not only do resources within a cluster install the VMs and connect to the grid quickly, the clouds do as well. While the results were similar, it should be noted that the time required to configure the static approach was not taken into effect. A process that is difficult to measure and is largely reliant on the ability of the administrator and the tools used. Whereas the time for the "Grid Appliance" does include many of these components.

It should be stated that the evaluation only has a single submission node. In a system with multiple submitters, the OpenVPN server could easily become a bandwidth bottleneck in the system as all data must pass through it, which 


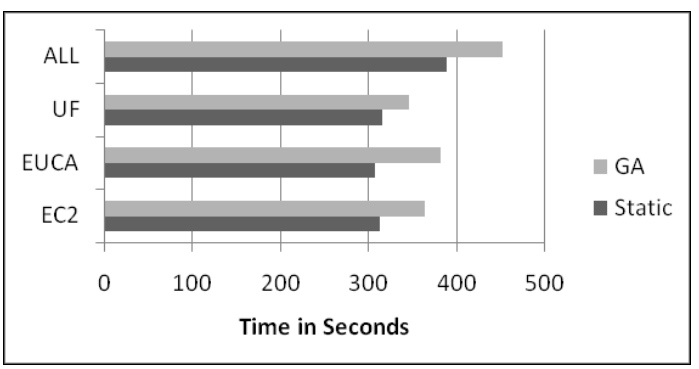

Figure 5: Comparison of times to run a 300 second job on each resource in various grids configured statically and through the "Grid Appliance." Legend: EC2 - Amazon's EC2, Euca - Indiana University's Eucalyptus, UF - University of Florida's ACIS Lab resources, Static - OpenVPN, GA - Grid Appliance.

can be avoided using IPOP. Additionally, the current "Grid Appliance" relies on polling with long delays, so as to not have negative effects on the system. Either shrinking those times or moving to an event based system should significantly improve the speed at which connectivity occurs.

\section{LESSONS LEARNED}

This section highlights some the interesting developments and experiences, we have had that do not fit the topics discussed so far.

\subsection{Deployments}

A significant component of our experience stems from the computational grid provided by Archer [27], an active grid deployed for computer architecture research, which has been online for over 3 years. Archer currently spans six seed universities contributing over $600 \mathrm{CPUs}$ as well as contributions and activities from external users. The Archer grid has been accessed by hundreds of students and researchers from over a dozen institutions submitting jobs totaling over 500,000 hours of job execution in the past two years alone.

The Grid Appliance has also been utilized by groups at the Universities of Florida, Clemson, Arkansas, and Northwestern Switzerland as a tool for teaching grid computing. Meanwhile the universities of Clemson and Purdue are using the Grid Appliance's VPN (GroupVPN / IPOP) to create their own grid systems. Over time, there have been many private, small-scale systems using our shared system available at www.grid-appliance.org with other groups constructing their own independent systems. Feedback from users through surveys have shown that non-expert users are able to connect to our public Grid appliance pool in a matter of minutes by simply downloading and booting a plug-and-play VM image that is portable across VMware, VirtualBox, and KVM.

\subsection{Towards Unvirtualized Environments}

Because of the demands put on Archer in terms of avoiding the overheads of virtualization and the perceived simplicity of managing physical resources as opposed to virtual resources running on top of a physical resources, many users have requested the ability to run Grid Appliances directly on their machine. Unlike clouds with machine imamges such as AMIs or VM appliances, physical machines images cannot be easily exported. Most physical OS installed on phyiscal machines will need some some custom tailoring to handle environment specific issues.

With this in mind, we moved away from stackable file systems and towards creating repositories with installable packages, such as DEB or RPM. The implications of packages mean that users can easily produce "Grid Appliances" from installed systems or during system installation. With the VPN router mode, mentioned earlier, resources in a LAN can communicate directly with each other rather than through the VPN. That means if they are on a gigabit network, they can full network speeds as opposed to being limited to $20 \%$ of that due to the VPN, overheads discussed in $[28]$.

\subsection{Advantages of the Cloud}

We have had the experience of deploying the "Grid Appliance" on three different cloud stacks: Amazon's EC2 [29], Future Grid's Eucalyptus [30], and Future Grid's Nimbus [31]. All of the systems, encountered so far, allow for data to be uploaded with each cloud instance started. The instance can then download the data from a static URL only accessible from within the instance, for example, EC2 user data is accessible at http://169.254.169.254/latest/user-data. A "Grid Appliance" cloud instances can be configured via user-data, which is the same configuration data used as the virtual and physical machines, albeit zip compressed. The "Grid Appliance" seeks the configuration data by first checking for a physical floppy disk, then in specific directory (/opt/grid \_appliance/var/floppy.img), followed by the EC2 / Eucalyptus URL, and finally the Nimbus URL. Upon finding a floppy and mounting it, the system continues on with configuration. Clouds have been also very useful for debugging. Though Amazon is not free, with Future Grid, grid researchers now have free access to both Eucalyptus and Nimbus clouds. Many bugs can be difficult to reproduce in small system tests or booting one system at a time. By starting many instances simultaneously, we have been able to quickly reproduce problems and isolate them, leading to timely resolutions, and verification of those fixes.

\subsection{Stacked File Systems}

Configuring systems can be difficult, which makes it important to have the ability to share the resulting system with others. The approach of actually creating packages can be overly complicated for novices. To address this concern, our original "Grid Appliance" supported a built-in mechanism to create packages through a stackable file system using copyon-write, as describe in [15]. In this environment, the VM used 3 disks: the "Grid Appliance" base image, the software stack configured by us; a module; and a home disk. In normal usage, both the base and module images are treated as read-only file systems with all user changes to the system being recorded by the home image, as depicted in Figure 6 .

To upgrade the system, users replaced their current base image with a newer one, while keeping their module and home disks. While the purpose of the module was to allow users to extend the configuration of the "Grid Appliance." To configure a module the system would be booted into developer mode, an option during the boot phase, where only the base and module images are included in the stacked file system. Upon completing the changes, a user would run a script that would clean the system and prepare it for shar- 


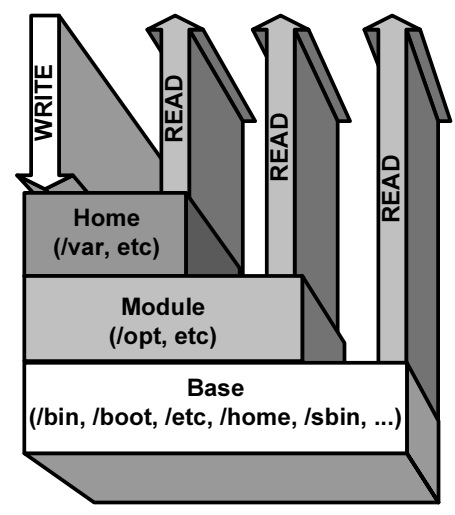

Figure 6: Example of a stackable file system from our previous "Grid Appliance." A file will be read from the top most file system in the stack and all writes are directed to Home.

ing. A user could then share the resulting module image with others.

Issues with this approach made it unattractive to continue using. First, there exists no kernel level support for stackable file systems, we had to add UnionFS [32] to the kernel, adding the weight of maintaining a kernel unto our shoulders. While FUSE (filesystem in userspace) solutions exist, they require modifications to the initial ram disk, which is reproduced automatically during the installation of every new kernel, furthermore, our experience with them suggests they are not well suited for production systems. Additionally, the approach was not portable to clouds or physical resources. So while we have deprecated the feature for now, we see it as a potential means to easily develop packages like DEB and RPM.

\subsection{Priority in Owned Resources}

In Archer, seed universities should have priority on the resources at their university. Similarly, users should have priority on their contributions. Otherwise, users will remove their resources from the grid, when they want guaranteed access. To support user and group based priorities, Condor has mechanisms that can be enforced at the server that allow for arbitrary means to specify user priority for a specific resource. So our configuration specifies that if the resource's user or group matches that of the submitter, the priority is higher than otherwise. This alone is not sufficient as malicious users could easily tweak their user name or group to obtain priority on all resources. Thus whenever this check is made the user's identity in the submission information is verified against their P2P VPN certificate. Failed matches are not scheduled and are stored in a log at the manager for the administrator to deal with later.

\subsection{Timing in Virtual Machines}

Certain applications, particularly license servers, are sensitive to time. Because of the nature of grids, there exist possibilities of having uncoordinated timing, such as improperly specifying the time zone or not using a network time protocol (NTP) server With regards to VMs, VMWare [33] suggests synchronizing with the host's time and to avoid using services like NTP, which may have adverse affects on timing inside the virtual machine. While NTP might have some strange behavior, relying on host time may produce er- ratic jumps in time that some software cannot handle. Our experiences recommends the use of NTP to address these concerns, which has resolved many issues with strange software behavior and frustration from users when their jobs fail due to being unable to obtain a license due to a timing mismatch.

\subsection{Selecting a VPN IP Address Range}

One challenge in deploying a VPN is ensuring that the address space does not overlap with that over the environments where it will be used. If there is overlap, users will be unable to connect to the VPN. Doing so will confuse the network stack, as there will be two network interfaces connected to the same address space but different networks. A guaranteed, though not necessarily practical solution is to run the resource on a VM NAT or a cluster NAT that does not overlap the IP address space of the VPN.

Users of the "Grid Appliance" should not have to concern themselves with this issues. Prior work on the topic by Ala Rezmerita et al. [34] recommends using the experimental address class E ranging between 240.0.0.0 - 255.255.255.254, unfortunately this requires Linux kernel modifications. With the amount of bugs and security fixes regularly pushed into the kernel, maintaining a forked kernel requires a significant amount of time, duplicating the work already being performed by the OS distribution maintainers. This would also limit the ability to easily deploy resources in physical and cloud environments. Additionally, users that wanted to multipurpose a physical resource may not want to run a modified kernel, while in most cloud setups the kernel choice is limited.

We have since moved towards using the 5.0.0.0 5.255.255.255 address range. Like the class E address space it is unallocated, but it requires no changes to any operating systems. The only limitation is that some other VPNs also use it, thus a user would not be able to run two VPNs on the same address space concurrently. This approach is much better than providing kernels or dealing with network address overlaps. Interestingly, even with this in place, we still see some "GroupVPNs" using address ranges in normal private network address ranges for the VPN, like 10.0.0.0 10.255.255.255 and 192.168.0.0 - 192.168.255.255.

\subsection{Administrator Backdoor}

While most administrators will agree that most problems that users encounter are self-inflicted, there are times, when the system is at fault. Debugging systems faults in a decentralized system can be very tricky, since it is very difficult to track down a resource in order to gain direct physical access. Additionally, having a user bring their resource to an administrator may be prohibitively complicated, as the user would need to relocate their "Grid Appliance" instance and have network connectivity in order to connect to the grid and show the problem to the administrator. To address this and other concerns that only appear after running the system for long periods of time, we have supplied an administrator backdoor into all resources by installing our public ssh key, though users are informed of this and are free to remove it for privacy concerns. In typical configurations, this approach might not be feasible, but because the "Grid Appliance" ships with a decentralized VPN supporting allto-all connectivity, any resource connected to the VPN is accessible for remote debugging by an administrator. Most 
users involved are extremely delighted with the process as it has an appearance that the system "just works."

\section{RELATED WORK}

Existing work that falls under the general area of desktop grids/opportunistic computing include Boinc [35], BonjourGrid [36], and PVC [34]. Boinc, used by many "@home" solutions, focuses on adding execute nodes easy; however, job submission and management rely on centralization and all tasks must use the Boinc APIs. BonjourGrid removes the need for centralization through the use of multicast resource discovery; the need for which limits its applicability to local area networks. PVC enables distributed, wide-area systems with decentralized job submission and execution through the use of VPNs, but relies on centralized VPN and resource management.

Each approach addresses a unique challenge in grid computing, but none addresses the challenge presented as a whole: easily constructing distributed, cross-domain grids. Challenges that we consider in the design of our system include allowing submission sites to exist any where without being confined to complex configuration or highly available, centralized locations; the ability to dynamically add and remove resources by starting and stopping a a resource; and the sharing of common servers so that no group in the grid is dependent on another. We emphasize these points, while still retaining the ease of use of Boinc, the connectivity of PVC, and the flexibility of BonjourGrid. The end result is a system similar to OurGrid [37]; however, OurGrid requires manual configuration of the grid and networking amongst sites, administration of users within a site, and limits network connectivity amongst resources, whereas "Grid Appliance" transparently handles these issues with a P2P overlay and VPN to handle network constraints and support network sandboxing and a web interface to configure and manage the grid.

With regards to clouds, there exists contextualization [38]. Users construct an XML configuration file that describes how a cloud instance should be configured and provide this to a broker. During booting of a cloud instance, it will contact a third-party contextualization broker to receive this file and configure the system. This approach has been leveraged to create dynamic grids inside the Nimbus cloud [39]. While this approach can reproduce similar features of the "Grid Appliance," such as creating grids inside the cloud, there are challenges in addressing cloud bursting, automated signing of certificates, and collaboration amongst disparate groups.

\section{CONCLUSIONS}

In this paper, we have presented a grid framework that enables users to easily deploy their own grids. By reducing the entry barrier to constructing wide-area grids, rather than just prividing a grid, our approach teaches users to create grids rather than providing access. The features of the "Grid Appliance" significantly simplify the construction of a grid over traditional approaches. These methods are based upon and have been verified through experience with individuals and groups coming from various backgrounds. Furthermore, we have presented both qualitative and quantitative utility of the "Grid Appliance" in Section 4. Namely, decentralized, P2P VPNs are resilient and easily configured; web interfaces ease the burden of crafting configuration files and signing of certificates; and package management systems can be used to create appliances nearly as conveniently as VMs. Those interested are able to test drive the system by coming to our public Web interface at the www.grid-appliance.org, where they can either use our public testing grid or deploy their own.

The concepts in this paper are intentionally generic so that they can easily be applied to other systems. For example, more complex approaches to grids involve entities known as virtual organizations. A virtual organization allows the same set of resources to be members of many distinct grids. Our web interface idea could be extended to support virtual organizations. Additionally, the sandboxing technique could be applied to many environments, including OurGrid, to allow grid network access without compromising the safety of the system.

For future work, we are considering mechanisms to fully decentralize the "Grid Appliance" by using a decentralized grid system that requires no manager nodes, though the challenges in doing so, are efficient resource discovery, clustering of group resources, and fair use scheduling. A completely decentralized grid could be constructed completely by client machines, in which, no one is more responsible than another for maintaining the grid.

\section{Acknowledgments}

This work is sponsored by the National Science Foundation (NSF) under awards 0751112 and 0721867. This material is based upon work supported in part by the NSF under grant 091812 (Future Grid). Any opinions, findings and conclusions or recommendations expressed in this material are those of the authors and do not necessarily reflect the views of the NSF.

\section{REFERENCES}

[1] M. Livny, J. Basney, R. Raman, and T. Tannenbaum, "Mechanisms for high throughput computing," SPEEDUP Journal, June 1997.

[2] Cluster Resources. (2010, July) Torque resource manager. http://www.clusterresources.com/pages/ products/torque-resource-manager.php.

[3] Sun. (2010, July) gridengine. http://gridengine.sunsource.net/.

[4] I. Foster, "Globus toolkit version 4: Software for service-oriented systems," Journal of Computer Science and Technology, vol. 21, pp. 513-520, 2006, 10.1007/s11390-006-0513-y. [Online]. Available: http://dx.doi.org/10.1007/s11390-006-0513-y

[5] P. Andreetto, et al., "The glite workload management system," Journal of Physics: Conference Series, vol. 119, no. 6, p. 062007, 2008.

[6] A. Ganguly, A. Agrawal, P. O. Boykin, and R. Figueiredo, "Wow: Self-organizing wide area overlay networks of virtual workstations," in IEEE High Performance Distributed Computing (HPDC), June 2006.

[7] P. O. Boykin and et al., "A symphony conducted by brunet," http://arxiv.org/abs/0709.4048, 2007.

[8] P. Maymounkov and D. Mazières, "Kademlia: A peer-to-peer information system based on the XOR metric," in International Workshop on Peer-to-Peer Systems (IPTPS), 2002. 
[9] G. DeCandia, D. Hastorun, M. Jampani, G. Kakulapati, A. Lakshman, A. Pilchin, S. Sivasubramanian, P. Vosshall, and W. Vogels, "Dynamo: amazon's highly available key-value store," in ACM SIGOPS symposium on Operating systems principles (SOSP). New York, NY, USA: ACM, 2007, pp. 205-220.

[10] A. Ganguly, D. Wolinsky, P. Boykin, and R. Figueiredo, "Decentralized dynamic host configuration in wide-area overlays of virtual workstations," in International Parallel and Distributed Processing Symposium, March 2007.

[11] D. I. Wolinsky, K. Lee, P. O. Boykin, and R. Figueiredo, "On the design of autonomic, decentralized vpns," in International Conference on Collaborative Computing: Networking, Applications and Worksharing, 2010.

[12] A. Ganguly and et al., "Improving peer connectivity in wide-area overlays of virtual workstations," in IEEE High Performance Distributed Computing (HPDC), 6 2008.

[13] A. Ganguly, A. Agrawal, P. O. Boykin, and R. Figueiredo, "IP over P2P: Enabling self-configuring virtual IP networks for grid computing," in International Parallel and Distributed Processing Symposium, 2006.

[14] D. I. Wolinsky, P. St. Juste, P. O. Boykin, and R. Figueiredo, "Addressing the P2P bootstrap problem for small overlay networks," in 10th IEEE International Conference on Peer-to-Peer Computing (P2P), 2010.

[15] D. I. Wolinsky and et al., "On the design of virtual machine sandboxes for distributed computing in wide area overlays of virtual workstations," in $V T D C, 2006$.

[16] R. J. Figueiredo, P. A. Dinda, and J. A. B. Fortes, "A case for grid computing on virtual machines," in ICDCS '03: Proceedings of the 23rd International Conference on Distributed Computing Systems. IEEE Computer Society, 2003.

[17] S. Santhanam, P. Elango, A. A. Dusseau, and M. Livny, "Deploying virtual machines as sandboxes for the grid," in WORLDS, 2005.

[18] K. Keahey, K. Doering, and I. Foster, "From sandbox to playground: Dynamic virtual environments in the grid," in Proceedings of 5th International Workshop in Grid Computing, November 2004.

[19] N. Andrade, L. Costa, G. Germoglio, and W. Cirne, "Peer-to-peer grid computing with the ourgrid community," in Proceedings of the 23rd Brazilian Symposium on Computer Networks, May 2005.

[20] D. H. J. Epema, M. Livny, R. van Dantzig, X. Evers, and J. Pruyne, "A worldwide flock of condors: Load sharing among workstation clusters," Future Generation Computer Systems, vol. 12, no. 1, pp. 53 65, 1996.

[21] LogMeIn. (2009) Hamachi. https://secure.logmein.com/products/hamachi2/.

[22] J. Yonan. (2009) OpenVPN. http://openvpn.net/.

[23] G. Sliepen. (2009, September) tinc. http://www.tinc-vpn.org/.
[24] X. Jiang and D. Xu, "Violin: Virtual internetworking on overlay," in Intl. Symp. on Parallel and Distributed Processing and Applications, 2003.

[25] M. Tsugawa and J. Fortes, "A virtual network (vine) architecture for grid computing," International Parallel and Distributed Processing Symposium (IPDPS), 2006.

[26] A. I. Sundararaj and P. A. Dinda, "Towards virtual networks for virtual machine grid computing," in Conference on Virtual Machine Research And Technology Symposium, 2004.

[27] R. J. Figueiredo, et al., "Archer: A community distributed computing infrastructure for computer architecture research and education," in Collaborative Computing: Networking, Applications and Worksharing, vol. 10. Springer Berlin Heidelberg, 2009, pp. 70-84. [Online]. Available: http://dx.doi.org/10.1007/978-3-642-03354-4\_7

[28] D. I. Wolinsky, Y. Liu, P. S. Juste, G. Venkatasubramanian, and R. Figueiredo, "On the design of scalable, self-configuring virtual networks," in IEEE/ACM Supercomputing, November 2009.

[29] (2009) Amazon elastic compute cloud. http://aws.amazon.com/ec2.

[30] D. Nurmi, R. Wolski, C. Grzegorczyk, G. Obertelli, S. Soman, L. Youseff, and D. Zagorodnov, "The eucalyptus open-source cloud-computing system," in IEEE/ACM International Symposium on Cluster Computing and the Grid (CCGrid), 2009.

[31] K. Keahey and T. Freeman, "Science clouds: Early experiences in cloud computing for scientific applications," in Cloud Computing and Its Applications, 2008.

[32] C. P. Wright and E. Zadok, "Unionfs: Bringing file systems together," Linux Journal, no. 128, pp. 24-29, December 2004.

[33] VMware, Inc., "Timekeeping in vmware virtual machines," http:

//www.vmware.com/pdf/vmware_timekeeping.pdf, 2008.

[34] A. Rezmerita, T. Morlier, V. Neri, and F. Cappello, "Private virtual cluster: Infrastructure and protocol for instant grids," in Euro-Par, 2006.

[35] D. P. Anderson, "Boinc: A system for public-resource computing and storage," in the International Workshop on Grid Computing, 2004.

[36] H. Abbes, C. Cérin, and M. Jemni, "Bonjourgrid: Orchestration of multi-instances of grid middlewares on institutional desktop grids," in International Parallel and Distributed Processing Symposium (IPDPS), 2009.

[37] N. Andrade, L. Costa, G. GermÃşglio, and W. Cirne, "Peer-to-peer grid computing with the ourgrid community," in Brazilian Symposium on Computer Networks (SBRC) - 4th Special Tools Session, 2005.

[38] K. Keahey and T. Freeman, "Contextualization: Providing one-click virtual clusters," in eScience, 2008.

[39] A. Harutyunyan, P. Buncic, T. Freeman, and K. Keahey, "Dynamic virtual AliEn grid sites on nimbus with CernVM," Journal of Physics: Conference Series, 2010. 